\title{
HYBRID OPTICAL SWITCHING IN OPTICAL CODE DIVISION MULTIPLEXING NETWORKS
}

\author{
P. Boobalan ${ }^{1}$, Mathimanirangan $\mathbf{M}^{2}$, Vinodh Kumar $\mathbf{V}^{3}$, Barakathulla $\mathbf{M}^{4}$ \\ 1, 2, 3, ${ }^{4}$ Department of Information Technology, Pondicherry Engineering College
}

\begin{abstract}
The amount of Internet traffic is dramatically increasing. Many high-bandwidth applications, such as teleconferencing, digital video broadcasting, and video-on-demand, rely on the Internet to carry multimedia traffic. The expanding Internet-based services are driving the need for a faster and better-service transmission technology in the Internet backbone. In this paper we are trying to implement hybrid optical switching which combines the three basic switching technologies such as optical circuit switching (OCS), Optical packet switching (OPS) and optical burst switching (OBS). Here the Wavelength division multiplexing is extended to optical code division multiplexing to increase the granularity. We have compared the QoS parameters of HOS in simple WDM and WDM/OCDM networks.
\end{abstract}

\section{INTRODUCTION}

Nowadays, the amount of Internet traffic is dramatically rising up. Many high-bandwidth real time applications like digital video broadcasting, video-on-demand and teleconferencing depend on the Internet to carry the multimedia traffic. So, the telephone companies now started offloading voice calls from public switched telephone networks to voice-over-Internet protocol (VoIP) networks for reducing the communication costs. The Internet is also firmly deep-seated in our daily lives. E-mail, instant messenger, social networks and web browsing have become substantial personal communication and information retrieval tools. The intensifying Internet-based services are driving the need for a faster and better-service transmission technology in the Internet backbone.

Wavelength division multiplexing (WDM) is a favorable transmission technology that can be used for the nextgeneration Internet. In WDM technology, the optical transmission spectrum is imprinted into a number of nonoverlapping wavelength bands, and each wavelength supports a single communication channel operating at the peak electronic rate. WDM can provide a large amount of raw bandwidth. Currently, WDM technology enables the multiplexing of 160-320 wavelengths into a single fiber, with a transmission rate of $10-40 \mathrm{~Gb} / \mathrm{s}$ per wavelength. There have also been numerous significant advances in optical components, such as fibers, amplifiers, laser sources, and filters, which have brought about more advanced WDM systems.

In parallel with the deployment in the WDM technology, the emergence of Internet applications, based upon the Internet protocol (IP), is causing a revolution in the telecommunication. It has become more and more clear that the common traffic convergence layer in the communication networks is going to be IP. The reason is that the ubiquitous $\mathrm{TCP} / \mathrm{IP}$ protocol is practically used in most end-user communications and many new applications.

Currently, there are three switching technologies in WDM networks, namely, optical circuit switching, optical packet switching, and optical burst switching. In this section, we discuss these three switching technologies.

In optical circuit switching, data traffic is delivered via circuits. A circuit or light path is set up from the source node to the destination node for each connection. The process of establishing a lightpath is as follows. When a connection request arrives, before data traffic is sent out, a control message is sent via an out-of-band control channel from the source node to the destination node in order to reserve wavelengths on links and establish a lightpath. If the lightpath is established successfully, an acknowledgement message is sent back via control channels to the source node. After the lightpath is set up, data traffic can then go through the WDM network all optically. After the data transmission is completed, the lightpath is released by a control message.

A lightpath may span multiple fiber links. In the absence of wavelength converters, a lightpath must occupy the same wavelength on all the fiber links through the network which it traverses. This property is known as the wavelength continuity constraint.

In optical circuit switching, once a lightpath is established, the lightpath will be dedicated to the corresponding connection. Hence, each connection has static wavelength allocation, which is suitable for smooth traffic (with constant bit rate), such as voice traffic. One advantage of optical circuit 
switching is that there is no data loss at the intermediate nodes. However, this switching technology may not be able to accommodate the highly variable and bursty nature of Internet traffic. Also, it takes a round trip time to set up a lighpath before data transmission, which results in long end-to-end delay. Furthermore, for the case of delivering a small amount of data traffic, having a dedicated lightpath is not efficient. Hence, this switching technology is justified only if a large amount of traffic needs to be transmitted.

The rapid increases in data traffic (bursty traffic) demands that the Internet is able to support bursty traffic and fast resource provisioning. Optical burst switching and optical packet switching are two promising methods for transporting bursty traffic directly over WDM networks, and which are capable of switching at Sub-wavelength granularities.

In Optical packet switching, data traffic is transmitted as photonic packets. Each packet consists of packet header and payload. Packet header and payload are sent out into the network together. At each optical switch, the packet header is processed electronically in order to configure the crossconnect for forwarding the packet to the next node, while the payload is delayed optically. This switching technology is similar to the store and forward manner in IP packet-switched networks. Since packets can arrive at the input ports of each node at different times, bit-level synchronization and fast clock recovery are required for packet header recognition and packet delineation. Also, when the packet header is processed, the payload needs to be delayed at fiber delay line. Due to the limited delay in fiber delay lines, the header processing time must be very small, which requires fast header processing speed. These stringent requirements make the optical packet switching difficult to implement using current available technologies.

A possible near-term alternative to optical packet switching is optical burst switching (OBS). In OBS networks, data traffic is transmitted as bursts. Each burst consists of multiple IP packets. A burst header packet is transmitted via control channels ahead of the corresponding burst in order to configure the switches along the burst's route. After an offset time, the burst cuts through the network all-optically via data channels. The offset time allows for the burst header packet to be processed and the switch to be set up before the burst arrives at the intermediate nodes; thus, no electronic or optical buffering is necessary at the intermediate nodes while the control packet is being processed. The burst header packet may also specify the duration of the burst in order to let the node know when it may reconfigure its switch for the next arriving burst. Hence, OBS networks are able to support dynamic bandwidth allocation and statistical multiplexing of data, efficiently utilizing WDM links.

OBS uses one-way signaling schemes, in which each data burst follows a corresponding burst header packet without waiting for an acknowledgment. A common signaling scheme for reserving resources in OBS networks is just-enough-time (JET), as shown in Fig. 1.1. By using this one-way based reservation scheme, the end-to-end delay experienced by data traffic is approximately one round-trip time less than that in optical circuit switching.

Table 1 Comparison of Three Optical Switching Technologies

\begin{tabular}{|l|l|l|l|l|l|l|}
\hline $\begin{array}{l}\text { Optical Switching } \\
\text { Technologies }\end{array}$ & $\begin{array}{l}\text { Bandwidth } \\
\text { utilization }\end{array}$ & $\begin{array}{l}\text { Setup } \\
\text { latency }\end{array}$ & $\begin{array}{l}\text { Switching } \\
\text { Speed Req. }\end{array}$ & $\begin{array}{l}\text { Processing } \\
\text { overhead }\end{array}$ & $\begin{array}{l}\text { Traffic } \\
\text { adaptivity }\end{array}$ & $\begin{array}{l}\text { Signaling } \\
\text { scheme }\end{array}$ \\
\hline Optical Circuit Switching & Low & High & Slow & Low & Low & Two way Out of band \\
\hline Optical Packet Switching & High & Low & Fast & High & High & One way In-band \\
\hline Optical Burst Switching & High & Low & Medium & Low & High & Two way Out of band \\
\hline
\end{tabular}

Table 1 summarizes the three optical switching technologies. From the figure, we can observe that optical burst switching has the advantages of both optical circuit switching and optical packet switching, while avoiding their shortcomings.

\section{NETWORK ARCHITECTURE OF HYBRID OPTICAL SWITCHING}

HOS networks consist of edge nodes and core nodes connected by WDM/OCDM links. The input traffic from multiple senders is assembled at the source edge node and is transmitted as bursts through high-capacity WDM/OCDM links over the optical core. A destination edge node, upon receiving a burst, disassembles the burst, and delivers the data packets to the corresponding receivers. Source edge nodes and destination edge nodes are called as ingress nodes and egress nodes respectively.

In HOS networks, data traffic is transmitted as bursts. Each burst consists of multiple packets (IP packets for an IP over WDM network), and a burst header packet (BHP) is generated for each burst. BHP contains the routing information to be used by core routers, the burst offset time, the data burst length, the data channel carrying the burst, and the QoS 
information. A burst and its BHP are transmitted separately on different channels. In a burst transmission scenario, a BHP is sent out ahead of the burst by an offset time in order to reserve resources at each intermediate core node along the path of the burst. While the BHP is processed at each intermediate node, the burst cuts through the pre-configured nodes all-optically. The offset time must ensure that, at each intermediate node, the

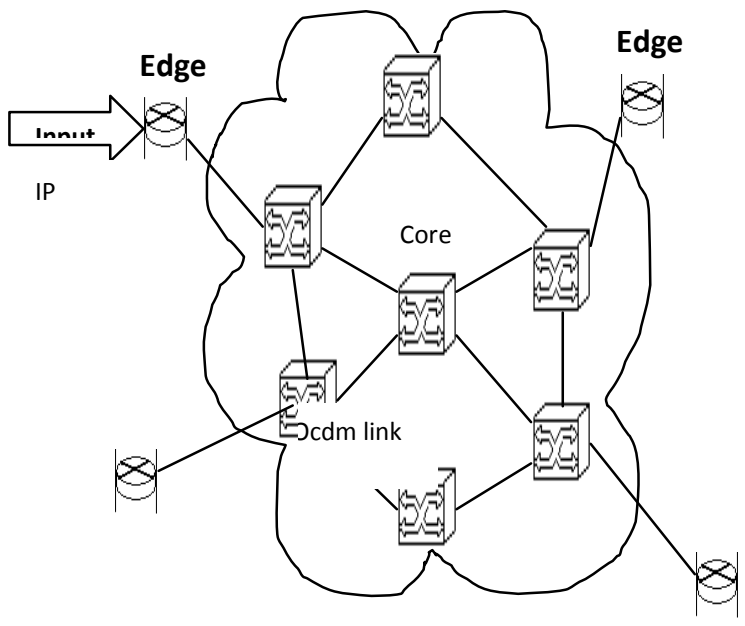

Fig 1 Network Architecture

BHP is processed prior to the burst arrival. Hence, there is no need to delay burst at each intermediate node.

\section{QUALITY OF SERVICE}

We have made a classification of the traffic services according to the loss, delayand the need for that service. By that way we have arrived at ten basic service categories they are telephones, signaling, video conferencing, real time interactive applications, streaming multimedia, video broadcasting, and data with low latency, data with low throughput, low priority data and standard data. As telephone and multimedia conferencing need continuous communication they are put under circuit switching likewise standard data and low throughout data are put in packets other services are sent through bursts.

\section{SIMULATION AND ANALYSIS}

\subsection{Simulation Setup}

We used network simulator-II in fedora operating system to simulate the whole network. The network involved fully optical networks and the links are changes as optical code division multiplexing links. A User Datagram Protocol agent is attached to certain nodes and respective sink agent is attached by the way the Transmission Control Protocol agents are also attached to certain nodes and respective sink agents are attached to certain nodes and some nodes are modified to form burst size of different values and the simulation is set to run in a network animator called NAM.

\subsection{QOS Parameters}

We took the basic two Qos parameters into consideration they are delay, and loss rates. Delay is the time taken by the packets to travel from source node to destination nodes in the packet and bursts for the circuit it is the time taken for the first connection establishment. The next parameter is the jitter which is the result of the difference between the maximum and minimum delay in the traffic units. The loss rate is the ratio between the number of packets sent to the number of packets received and the number of burst formed to the number of burst received and for circuits the loss is the ratio between the number of requests made to establish a circuit and the number of circuits rejected.

For calculating the delay we used some formulas taking into consideration the factors which involves in the delay of a packet.

The formulas are as follows.

- Delay for packets.

$$
\mathrm{Dp}=\text { Dra }+ \text { Dprop }
$$

- Delay for bursts.

$$
\mathrm{Db}=\text { Dassem }+ \text { Dra }+ \text { Dprop }
$$

- Delay for circuits.

$$
\mathrm{Dc}=\text { Dprop }
$$

The Dra is the delay made by resource allocator and the Dassem is the delay made by the assembler.

\section{RESULTS}

The values are calculated properly and plotted in the graph for better comparison with the normal WDM HOS networks and the WDM/OCDM HOS networks.

\subsection{Delay}

The delay is calculated as per the formula mentioned in the earlier part the circuits are neglected in this parameter as it involves only the propagation delay and it will be good when the granularity is increased there will be a separate slot for the traveling of packets in every link for this circuit. And the graphs are plotted with four traffic units such as WDM packet, WDM burst, OCDM packet and OCDM burst. WDM is the normal

Hos network proposed [1]. OCDM units are the new ones. The graph shows all the four paramters comes as follows. 


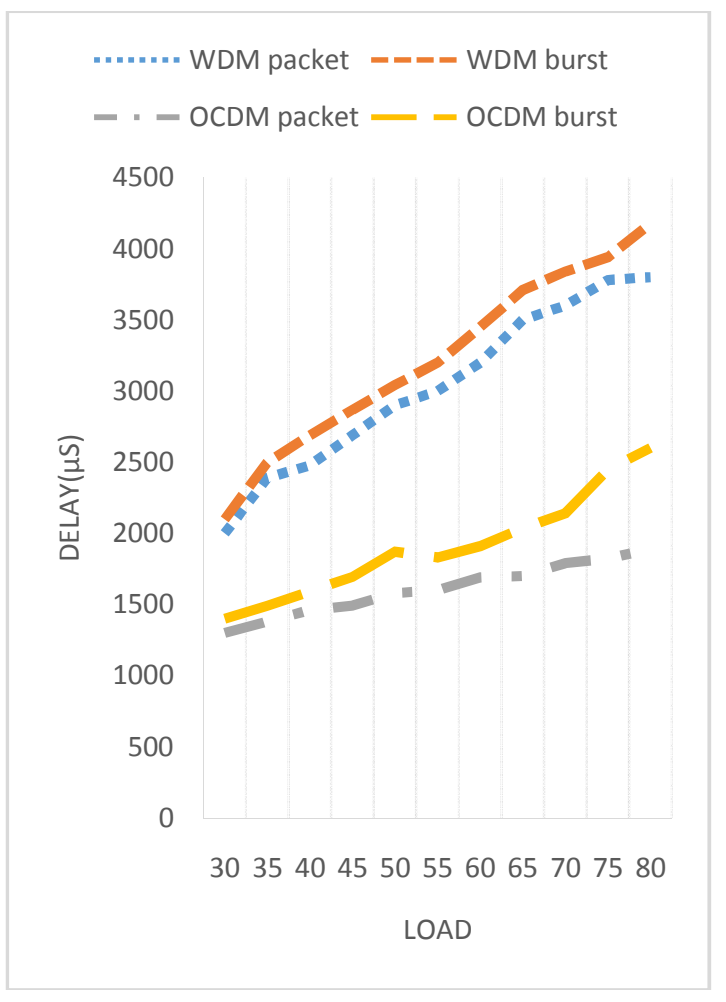

Fig 2 Graph showing the delay of each traffic units based on the load.

From this it is evident that OCDM network traffics units experience a very less delay when compared to the normal WDM networks. In OCDM networks also the burst experience a bit higher delay when compared to the packets due to their size. And there is possibility that the OCDM bursts may meet WDM packets when the load is increased a lot. The next parameter taken into consideration is loss rate.

\subsection{Loss Rate.}

The loss rates are calculated for the packets as the ratio between the packets send and packets lost, for the burst as bursts generated to the bursts lost and for the circuits as the request for the circuit to be formed and the circuit request rejected. The values are calculated and plotted in graph and the graph comes as follows.

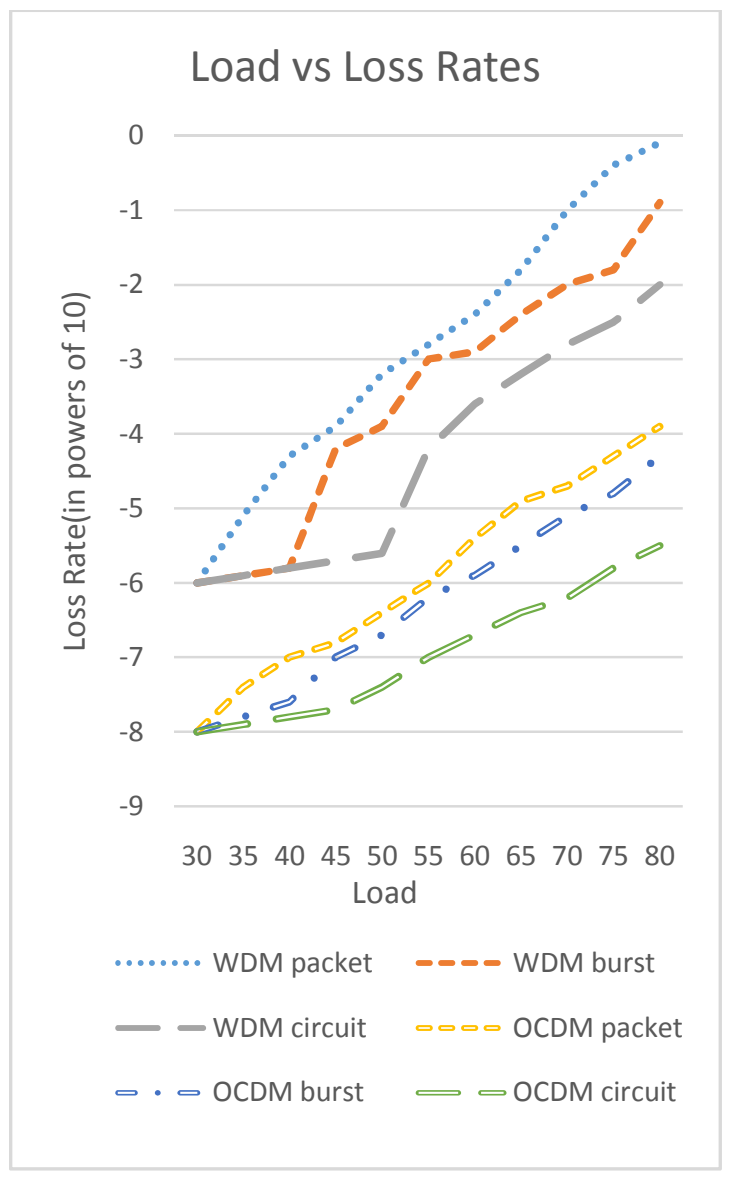

Fig 3 Graph showing the loss rates of different traffic units with respect to the load in that network.

From the graph it is evident that the circuits have less probability of loss in both in the WDM and the OCDM networks but it is very less in the OCDM networks than the WDM networks. These are the two metrics used to compare the normal WDM based HOS network with the OCDM based HOS network the other parameters are energy efficiency and power consumption but as the network simulator does not provide facilities for that kind of calculations but with these two parameters it is evident that that OCDM networks are better than HOS networks.

\section{CONCLUSIONS}

In this paper we analyzed a novel HOS network architecture that efficiently combines three different switching paradigms, namely, circuit, burst, and packet switching, on the same network.. The most appropriate switching method is selected for the transmission of traffic generated by different applications, ensuring efficient QoS differentiation at the optical layer. Performance of the propose network is simulated by the network simulator and the results are tabulated and plotted in graph and compared with normal HOS network to show that OCDM better than that. Although the values are not 
that much differs shows a better network can be defined to improve the transmission and switching a lot better. The main differences between the two networks is the loss rates in the circuits and delay in packets as there is always a slot allotted for transmission the loss rate in the circuit is very less and the delay for the packet transmission is very less when compared to the WDM based HOS networks.

In future many switching technologies may come and this technology can be replaced but this network is better than the normal HOS network.

\section{REFERNCES}

[1] Matteo Fiorani, Maurizio Casoni, and Slavisa Aleksic,"Hybrid Optical Switching for Energy Efficiency and QoS Differentiation in Core Networks", j. opt. commun. netw./vol. 5, no. 5/may 2013.

[2] Hamzeh Beyranvand Jawad A. Salehi, "Efficient Optical Resource Allocation and QoS Differentiation in Optical Burst Switching Networks Utilizing Hybrid WDM/OCDM", journal of lightwave technology, vol. 30, no. 15, august 1, 2012.

[3] Cisco white paper, "Cisco Visual Networking Index: Forecast and Methodology, 2011-2016," May 2012.

[4] T. Miyazawa, H. Furukawa, K. Fujikawa, N. Wada, and H.Harai, "Development of an autonomous distributed control system for optical packet and circuit integrated networks," J. Opt. Commun. Netw., vol. 4, no. 1, pp. 25-37, Jan. 2012.

[5] J. Baliga, R. Ayre, K. Hinton, W. Sorin, and R. S. Tucker, "Energy consumption in IP optical networks," J. Lightwave Technol., vol. 27, no. 13, pp. 2391-2403, July 2009.

[6] "Definition of the differentiated services field (DS Field) in the IPv4 and IPv6 headers," IETF RFC 2474, Dec. 1998.

[7] "An architecture for differentiated services," IETF RFC 2475, Dec. 1998.

[8] S. Aleksic, "Analysis of power consumption in future high-capacity network nodes," J. Opt. Commun. Netw., vol. 1,no. 3, pp. 245-258, Aug. 2009.

[9] D. J. Blumenthal, J. Barton, N. Beheshti, J. E. Bowers, E.Burmeister, L. A. Coldren, M. Dummer, G. Epps, A. Fang,Y. Ganjali, J. Garcia, B. Koch, V. Lal, E. Lively, J. Mack, M.Masanovic, N. McKeown, K. Nguyen, S. C. Nicholes, H. Park,B. Stamenic, A. Tauke-Pedretti, H. Poulsen, and M. Sysak, "Integrated photonics for low-power packet networking,"J. Sel. Top. Quantum Electron., vol. 17, no. 2, pp. 458471,Mar./Apr. 2011.

[10] R. Cafini, W. Cerroni, C. Raffaelli, and M. Savi, "Standardbasedapproach to programmable hybrid networks," IEEECommun. Mag., vol. 49, no. 5, pp. 148-155, May 2011.
[11] N. Stol, M. Savi, and C. Raffaelli, "3-level integrated hybrid optical network (3LIHON) to meet future QoS requirements," in IEEE Global Telecommunications Conference (GLOBECOM 2011), Houston, TX, Dec. 5-9, 2011.

[12] Y. Chen, C. Qiao, and X. Yu, "Optical burst switching: A new area in optical networking research," IEEE Netw., vol. 18, no. 3, pp. 16-23,May 2004.

[13] S. J. Ben Yoo, "Optical packet and burst switching technologies for the future photonic internet," J. Lightw. Technol., vol. 24, no. 12, pp.4468-4492, Dec. 2006.

[14] T. Venkatesh and C. S. R. Murthy, An Analytical Approach to Optical Burst Switched Networks. New York: Springer-Verlag, 2010. 